\title{
Investigación en Tecnología Educativa y avance del conocimiento abierto
}

\author{
Jesús Salinas \\ Universidad de las Islas Baleares \\ jesus.salinas@uib.es
}

La investigación en Tecnología Educativa, desde siempre se ha situado en el contexto de la sociedad digital, respondiendo a las tres características que para Weller (2011) presentan las nuevas prácticas académicas: digitales, en red y abiertas. Sin embargo, en temas de la construcción, intercambio y comunicación del conocimiento parece ir doblegándose a las tendencias que se dan en la academia.

Hace poco, Salinas y Marin (2019) publicábamos un trabajo sobre colaboración científica e identidad digital académica en redes sociales, y comenzábamos reflexionando sobre cómo la creciente digitalización de las prácticas de los investigadores presenta diversos ámbitos de atención. Nuestro foco estaba dirigido hacia las redes sociales académicas y su papel en el avance del conocimiento, pero aparecen otros temas que a mi juicio requieren reflexión por parte de los propios investigadores en relación a la contribución de cada uno de nosotros al avance y el intercambio del conocimiento científico: el acceso abierto a dicho conocimiento y las políticas editoriales, institucionales y personales de todo tipo en relación a ello, la puesta a disposición de los trabajos publicados o en preprint en las redes académicas al uso, la investigación en colaboración y la coautoría, los repositorios institucionales y temáticos, etc..

Este no es el lugar para iniciar una reflexión en toda regla sobre los nuevos enfoques acerca de la construcción y comunicación del conocimiento científico, pero si que podemos llamar la atención sobre algunas de las tendencias a que hacíamos referencia, al menos para tenerlas presentes.

La primera y más grave, a mi entender, es el del acceso abierto al conocimiento. Con ser un tema muy complejo hay motivos de preocupación y reflexión. Las tendencias en el ámbito de las publicaciones científicas nos están acostumbrando a un cierto "acceso abierto" que no es tal, dado que muchas de estas revistas pertenecen a entramados empresariales que sí tienen ánimo de lucro, y que lo que está ocurriendo es que se ha trasladado el costo del lector al investigador que publica, todo ello agravado por la penalización que para los investigadores de países en vías de desarrollo supone.

Obviamente, encontramos un enorme listado de publicaciones que se constituyen como espacios de participación activa, de presencia y socialización del conocimiento, pero que en muchos casos sin el trabajo de apoyo voluntario no podrían funcionar. Pongamos por caso esta misma revista, RIITE. Este tipo de revistas abiertas, de construcción colaborativa y voluntaria por distintas razones van dejando de ser atractivas para que los investigadores las contemplen como vías de comunicación de sus trabajos (impacto medido de las revistas, temas de rendición de cuentas de méritos de investigación, etc..).

Pero los grandes grupos de presión que rigen la industria editorial científica, condicionan los temas, los productos y los procedimientos de investigación. También impactan en la investigación colaborativa al condicionar la coautoría penalizada si es de la misma institución o del mismo país y promoviendo coautoría internacional que en algunos casos resulta artificial. 
Esto hace que cada vez los artículos, acomodados a las tendencias, sean más adecuados formalmente -adecuados a los cánones establecidos- independientemente de la calidad de los productos de investigación que describen. Creo que una buena investigación para confirmar esto podría ser analizar los argumentos de rechazo de artículos por parte de editores en primera instancia. Sería difícil lograr dichos argumentos de las mismas revistas y supongo que también de los investigadores, pero sin duda sería altamente ilustrativo.

Otra forma de condicionar se puede observar a través de medidas de rendición de cuentas del reconocimiento investigador que dependen en gran medida de las métricas convencionales de producción de conocimiento (por ejemplo, número de publicaciones y citas y tipo de revistas académicas), en gran parte alejadas del acceso abierto.

Las redes sociales académicas contribuyen de forma significativa a promocionar y mantener una identidad digital académica ofreciendo una plataforma para que los investigadores se presenten a sí mismos y a sus investigaciones, dando acceso a documentos científicos.

La difusión del conocimiento experimenta profundos cambios en estos días debido a la importancia que estas redes adquieren en relación a la divulgación, la promoción del trabajo de los investigadores, la cooperación e intercambio de conocimiento, etc... Pero por otra parte, no vale ya lograr que un trabajo de investigación llegue a una publicación científica. El investigador debe promocionar su trabajo en dichas redes ya sea por motivación propia o por presión de las propias revistas.

Todas estas facilidades pueden dar la idea a los investigadores de encontrarse en un ambiente de acceso abierto a la investigación. Al igual que pasa con muchas de las redes sociales digitales, ResearchGate y Academia.edu., p.e., son compañías comerciales y no permiten a sus usuarios tomar sus propios datos y reutilizarlos en ningún otro lugar, ni sus términos de servicio permiten fácilmente extraer esos datos en nombre de los autores para incorporarlos, digamos, a un repositorio institucional o temático (Salinas y Marín, 2019).

Podemos encontrar otros muchos aspectos controvertidos a propósito de la comunicación del conocimiento. El avance de las tecnologías digitales facilita y promueve, a la vez que incorpora prácticas controvertidas. Esperamos que los trabajos incluidos en este número contribuyan a la mirada crítica necesaria sobre ellos.

Este número 6 de la Revista RIITE incluye como primera colaboración, una entrevista con Catherine Cronin, Desarrolladora de Educación Estratégica en el Foro Nacional para la Mejora de la Enseñanza y el Aprendizaje en la Educación Superior en Irlanda, donde nos habla sobre prácticas de educación abierta en Educación Superior y reflexiona sobre algunas cuestiones clave en torno a la misma ¿por qué ha de ser abierta la educación? ¿quién/es son los responsables? y ¿cuáles son las implicaciones?

Incluye, además, un trabajo de Patrícia B. Scherer Bassani (Feevale University) e llona Buchem (Beuth University of Applied Sciences), sobre como el intercambio virtual puede definirse como una forma de movilidad virtual para ampliar el alcance y ámbito de los programas tradicionales de aprendizaje intercultural. Este artículo presenta un ejemplo de un intercambio virtual llamado InterCult - Competencias Interculturales - que pretendía dar la oportunidad a los estudiantes de Alemania, Francia y Brasil de explorar aspectos interculturales a través de la colaboración en línea.

El siguiente trabajo de Anna Escofet, Begoña Gros, Marta López Costa (Universitat de Barcelona) y Marta Marimon-Martí (Universitat de Vic - Universitat Central de Catalunya), se ocupa de la percepción docente y muestra los resultados de una investigación que tiene por objetivo analizar los conocimientos, preferencias, percepciones y necesidades del profesorado respecto a la configuración del aula como espacio de aprendizaje, concretamente en relación a la dimensión digital. Para ello se realizó un estudio cuantitativo mediante el método de encuesta a una muestra de 847 docentes de educación infantil, primaria y secundaria. Los resultados obtenidos muestran que los docentes perciben que la dimensión digital debería ser uno de los elementos clave para plantear nuevos espacios de aprendizaje en las escuelas.

Otro de los trabajos incluídos lo presenta Ana Belén Villarejo Villar, del IES Castillo de la Yedra de Cazorla y se ocupa de la realidad aumentada en la enseñanza de ciclos formativos 
mediante el diseño, implementación y evaluación de una propuesta didáctica basada en la realidad aumentada para la materia de anatomorfisiología en los ciclos de grado medio de Cuidados Auxiliares de Enfermería y de Emergencias Sanitarias. Para ello se recoge información sobre cuatro grandes dimensiones como son la atención, la confianza, la relevancia y la satisfacción relacionadas con la experiencia

Y por último, se incluye un trabajo de Marta Gallardo (Universidad de Murcia) y Marta JordiTaltavull (Johannes Gutenberg Universität Mainz), donde presentan una experiencia en un entorno universitario mediante el análisis de conceptos de la Geografía Económica a través del visionado de videoclips musicales integrados en la plataforma YouTube.

Completa el número la recensión de Maa Ángeles Hernández Prados (Universidad de Murcia) sobre García Fernández, F., y Sala, X. B. (2007). Educar hijos interactivos. Madrid, Instituto de Ciencias para la Familia, universidad de Navarra, obra originaria ampliamente versionada y adaptada en posteriores ediciones para describir la realidad de otros países latinoaméricanos. La obra aglutina diversos aspectos relacionados con el debate ético-social imperante en ese momento, convirtiendo a los autores en un referente a citar respecto a la educación de las nuevas generaciones ante el uso que hacen de las TIC.

Al analizar estos trabajos y cualquier publicación de investigaciones, conviene no olvidar, como señalan Cummings, Regeer et al. (2018) al analizar los discursos que se efectúan sobre la justificación de las tecnologías en la Sociedad de la Información y el Conocimiento (SIC), que suelen darse dos propuestas para su justificación que tienen cargas ideológicas diferentes: a) un discurso tecno-científico-económico, en gran parte propuesto por gobiernos de países desarrollados, que plantea que el conocimiento científico y tecnológico condiciona directamente el desarrollo económico y social de las sociedades modernas, lo que le otorga un marcado carácter determinista y estandarizador, y b) otro más pluralista-participativo que tiende a otorgarles un rol más inclusivo de las mismas en la sociedad, favoreciendo los saberes locales y al desarrollo endógeno y significativo de la persona y máximo respeto por la diversidad lingüística y cultural.

Esperamos que los trabajos que presentamos, lo mismo que la propia revista, contribuyan al avance del conocimiento y del acceso abierto y transparente al mismo desde esta segunda perspectiva. Por ello, felicitamos a todos los autores que desinteresadamente comparten aquí sus investigaciones, con la esperanza que los avances que nos presentan contribuyan al conocimiento abierto en el campo de la Tecnología Educativa.

\section{REFERENCIAS BIBLIOGRÁFICAS}

Cummings, S., Regeer, B., de Haan, L., Zweekhorst, M., y Bunders, J. (2018). Critical discourse analysis of perspectives on knowledge and the knowledge society within the Sustainable Development Goals. Development Policy Review, 36, 727-742.

Salinas, J. y Marin, V.I. (2019). Metasíntesis cualitativa sobre colaboración científica e identidad digital académica en redes sociales. Revista Iberoamericana de Educación a Distancia, 22(10), 97-117.

Weller, M. (2011). The digital scholar: How technology is changing academic practice (1st ed.). London: Bloomsbury Publishing PLC. 


\section{INFORMACIÓN SOBRE EL AUTOR}

\section{Jesús Salinas}

Universidad de las Islas Balerares

Doctor en Filosofía y Ciencias de la Educación. Catedrático de Tecnología Educativa de la Universidad de las Islas Baleares. Investigador Principal del Grupo de Tecnología Educativa. Coordinador del Doctorado Interuniversitario en Tecnología Educativa. Director del Máster en Tecnología Educativa: elearning y gestión del conocimiento. Director de Edutec - Revista Electrónica de Tecnología Educativa

\section{cc) (i) (-)}

Los textos publicados en esta revista están sujetos a una licencia de Reconocimiento 4.0 España de Creative Commons. Puede copiarlos, distribuirlos, comunicarlos públicamente y hacer obras derivadas siempre que reconozca los créditos de las obras (autoría, nombre de la revista, institución editora) de la manera especificada por los autores o por la revista. La licencia completa se puede consultar en:Licencia Creative Commons Atribución-NoComercial-Compartir por igual 4.0 Internacional. 